\title{
Caprine MAVS Is a RIG-I Interacting Type I Interferon Inducer Downregulated by Peste des Petits Ruminants Virus Infection
}

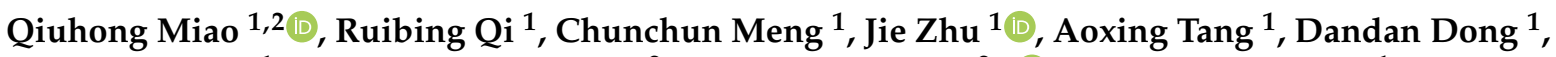 \\ Hongyuan Guo ${ }^{1}$, Monique M. van Oers ${ }^{2}$, Gorben P. Pijlman ${ }^{2, *}$ and Guangqing Liu ${ }^{1, *}$ \\ 1 Innovation Team of Small Animal Infectious Disease, Shanghai Veterinary Research Institute, \\ Chinese Academy of Agricultural Science, Shanghai 200241, China; qiuhong.miao@wur.nl (Q.M.); \\ gqyzqrb@gmail.com (R.Q.); mengcc@shvri.ac.cn (C.M.); zj121@shvri.ac.cn (J.Z.); tax1366@163.com (A.T.); \\ dddong2015@126.com (D.D.); guohy9510@163.com (H.G.) \\ 2 Laboratory of Virology, Wageningen University \& Research, Droevendaalsesteeg 1, \\ 6708 PB Wageningen, The Netherlands; monique.vanoers@wur.nl \\ * Correspondence: gorben.pijlman@wur.nl (G.P.P.); liugq@shvri.ac.cn (G.L.)
}

check for updates

Citation: Miao, Q.; Qi, R.; Meng, C.; Zhu, J.; Tang, A.; Dong, D.; Guo, H.; van Oers, M.M.; Pijlman, G.P.; Liu, G. Caprine MAVS Is a RIG-I Interacting Type I Interferon Inducer Downregulated by Peste des Petits Ruminants Virus Infection. Viruses 2021, 13, 409. https://doi.org/ 10.3390/v13030409

Academic Editor: Satya Parida

Received: 1 December 2020

Accepted: 1 March 2021

Published: 5 March 2021

Publisher's Note: MDPI stays neutral with regard to jurisdictional claims in published maps and institutional affiliations.

Copyright: (c) 2021 by the authors. Licensee MDPI, Basel, Switzerland. This article is an open access article distributed under the terms and conditions of the Creative Commons Attribution (CC BY) license (https:// creativecommons.org/licenses/by/ $4.0 /)$.

\begin{abstract}
The mitochondrial antiviral-signaling protein (MAVS, also known as VISA, IPS-1, or CARDIF) plays an essential role in the type I interferon (IFN) response and in retinoic acid-inducible gene I (RIG-I) mediated antiviral innate immunity in mammals. In this study, the caprine MAVS gene (caMAVS, $1566 \mathrm{bp}$ ) was identified and cloned. The caMAVS shares the highest amino acid similarity (98.1\%) with the predicted sheep MAVS. Confocal microscopy analysis of partial deletion mutants of caMAVS revealed that the transmembrane and the so-called Non-Characterized domains are indispensable for intracellular localization to mitochondria. Overexpression of caMAVS in caprine endometrial epithelial cells up-regulated the mRNA levels of caprine interferon-stimulated genes. We concluded that caprine MAVS mediates the activation of the type I IFN pathway. We further demonstrated that both the CARD-like domain and the transmembrane domain of caMAVS were essential for the activation of the IFN- $\beta$ promotor. The interaction between caMAVS and caprine RIG-I and the vital role of the CARD and NC domain in this interaction was demonstrated by co-immunoprecipitation. Upon infection with the Peste des Petits Ruminants Virus (PPRV, genus Morbillivirus), the level of MAVS was greatly reduced. This reduction was prevented by the addition of the proteasome inhibitor MG132. Moreover, we found that viral protein V could interact and colocalize with MAVS. Together, we identified caMAVS as a RIG-I interactive protein involved in the activation of type I IFN pathways in caprine cells and as a target for PPRV immune evasion.
\end{abstract}

Keywords: caprine; mitochondrial antiviral signaling protein (MAVS); Peste des Petits Ruminants Virus (PPRV); innate immunity

\section{Introduction}

The induction of the interferon (IFN) response is vital for vertebrate hosts to counteract invading viruses and microbial pathogens. In general, cytoplasmic pathogen-associated molecular patterns (PAMPs), such as dsRNA molecules generated during viral infection, are sensed by host pattern recognition receptors, which include toll-like receptors (TLRs), and retinoic-acid inducible gene I (RIG-I)-like receptors (RLRs) and nucleotide-binding oligomerization domain-like receptors (NLRs) [1]. Once invading pathogens are recognized, these receptors activate downstream signal transduction pathways.

RLRs constitute a critical group of intracellular viral RNA sensors that share similar structures and functions: RIG-I, melanoma differentiation-associated gene 5 (MDA5), and Laboratory of Genetics and Physiology 2 (LGP2). RLRs contain an ATP-dependent RNA helicase domain that is required for double-stranded RNA binding and ATP hydrolysis [2]. RLRs share the ability to detect distinct viral RNA structures as it has been shown for many RNA viruses, including retrovirus, influenza virus and Sendai virus, and for some DNA 
viruses, such as Epstein-Barr virus, and adenovirus [3]. RIG-I and MDA5 each possess two $\mathrm{N}$-terminal tandem caspase activation and recruitment domains (CARDs) that mediate the signaling to Mitochondrial Antiviral Signaling protein (MAVS), leading to the activation of IFN-mediated antiviral immunity. LGP2, on the other hand, lacks CARDs and is thought to have a regulatory function [4].

MAVS functions as the central signaling protein in the RLR-mediated innate immunity pathway and signals to downstream cytokine production, including type I IFN and ultimately IFN-stimulated genes (ISGs). MAVS is also referred to in the literature as IPS-1, VISA and CARDIF [5-8], and orthologs have been found in several vertebrate species such as mouse [9], tree shrew [10], cats [11], and goose [12], but also in mollusc [13]. The deletion of MAVS has been shown to significantly reduce the levels of antiviral and other pro-inflammatory cytokines normally induced by virus infection [14]. The central role of MAVs in host antiviral immunity is further illustrated by the fact that double-knockout mice (MAVS- / - ) are highly susceptible to multiple RNA virus infections [15].

Structurally, MAVS contains an N-terminal CARD-like region, a Proline-Rich region (PRR), and a C-terminal transmembrane (TM) domain, of which the CARD-like and TM domains have been demonstrated to be essential for MAVS signalling in human cells [5]. Initially, MAVS was reported to predominantly localize in mitochondria, where it maintains the stability and function of these organelles [5]. In addition, MAVS was detected in peroxisomes. Upon viral infection, peroxisomal MAVS provides short-term protection by rapidly inducing IFN-independent expression factors, whereas mitochondrial MAVS has been associated with the activation of a more stable antiviral response involving IFN production [16]. During viral infection, the cellular localization of MAVS may change. It has been shown that, following infection with several RNA viruses, mitochondrial-associated endoplasmic reticulum membrane (MAM) was recruited by RIG-I, which then bound to MAVS, and in that way, coordinated the cellular localization of MAVS to regulate the activation of an effective antiviral response [17]. Functional differences among mitochondria MAVS, peroxisomal MAVS, and MAM localized MAVS suggest that the MAVS protein could recruit distinct cellular components, depending on its cellular localisation and as a consequence may form a powerful network during the innate immune response [18].

Viruses have been shown to apply different strategies to counteract MAVS-mediated signaling, and as a consequence, evade the associated innate immune responses. An example is the hepatitis $\mathrm{C}$ virus (HCV) NS3/4A protease, which localizes between the MAM and mitochondria, and particularly targets MAVS that is associated with MAM. Since MAM-localized MAVS has been recognized as being capable of transducing RIG-I signalling, HCV NS3/4A protease is predicted to interrupt RIG-I mediated signalling by cleaving MAVS [17].

Thus, MAVS is a central protein in the induction of type I IFNs in a number of mammals, but data for goats are missing thus far. Infections with the morbillivirus, Peste des Petits Ruminant Virus (PPRV, family Paramyxoviridae, genus Morbillivirus) are characterized by conjunctivitis, rhinitis, pneumonia and stomatitis, and enteritis, and reach mortalities as high as $90 \%$ in small ruminants. PPRV replication occurs in the respiratory tract, where antigen-presenting cells (APC) (or dendritic cells (DC), macrophages and monocytes) are infected and lymphocytes play a major role during immune responses [19]. PPRV causes immunosuppression in its natural hosts. The virus counteracts the IFN response and signalling pathways. Even though IFNs and ISGs are important during innate immune response, PPRV controls the induction of type I IFNs [20]. To better understand the pathogenesis of PPRV infection and other caprine-related infectious diseases we aimed to characterize the function of caprine (ca) MAVS as a central protein during the innate immune response in caprine cells and analyse its role in type I IFN induction. Furthermore, we investigated whether PPRV infection affected caMAVS levels and whether specific viral proteins interacted with caMAVS. We found that the viral protein $\mathrm{V}$ could interact and colocalize with MAVS. By expanding our insight in the mode of action of PPRV in the 
suppression of innate immunity, we aim to contribute to the control of viral diseases of goats, in particular in PPRV and related morbillivirus infections.

\section{Materials and Methods}

\subsection{Cloning and Sequence Analysis of Caprine MAVS}

Total RNA from immortalized caprine endometrial epithelial cells (EECs) was extracted by using TRIzol reagent (Invitrogen, Carlsbad, CA, USA). Total RNA concentration, as well as the $260 / 280$ and 260/230 ratio, were measured by spectrophotometer (Biotek, Winooski, VT USA). Then cDNA was generated by using M-MLV reverse transcriptase (Promega, USA) with random primers (Invitrogen). Primers (Supplementary Materials Table S1) were designed for amplification of caMAVS and caRIG-I based on the predicted sequence in GenBank (accessions XM_018057391.1 and XM_005683566.3). PCR amplification was performed in a reaction mixture of $50 \mu \mathrm{L}$ containing TransStart ${ }^{\circledR}$ FastPfu Fly DNA polymerase (Transgen Biotech, Beijing, China). The PCR products were purified on spin columns from Sangon Biotech (Shanghai, China) and cloned to $\mathrm{pEASY}^{\circledR}{ }^{\circledR}$-BLUNT Zero Vector (Transgene Biotech, China). Inserts of five positive clones were sequenced (Sangon Biotech, Shanghai, China). To analyze the evolution of caprine MAVS, a phylogenetic tree was constructed based on the DNA coding sequence of MAVS proteins from 19 animal species selected as listed in Table S2. The phylogenetic tree was built with the neighborjoining method with 1000 bootstrap replications by MEGA5.0. The amino acid sequence of caMAVS and those of MAVS from other species, such as human, rabbit, mouse, and sheep, were aligned using the MEGA 5.0 program and edited by the MegAlign package of Lasergene.

\subsection{Construction of Expression Plasmids}

Expression plasmids of Myc-tagged caMAVS and caRIG-I were constructed by inserting the full-length open reading frames (ORFs) into the pCMV-MYC vector with MYC fused to the N-terminus (Clontech, Takara Bio, Kusatsu, Shiga, Japan). HA and $3^{*}$ Flag-tagged caMAVS constructs were cloned with Infusion technology (Vazyme Biotech Co., Ltd., China) into pCMV-HA (N-Terminal) (Clontech, Japan) and p3*FLag-CMV-10 (Sigma-Aldrich, USA) vectors, respectively. Truncated forms of caMAVS, which lacked the CARD domain (residues 10-77), PRR domain (residues 78-199), Transmembrane (TM) domain (residues 495-517), or Non-Characterized (NC) domain (residues 201-494) were constructed by using Infusion technology or overlapping extension PCR, as described previously [21]. Flag-tagged human MAVS (huMAVS) plasmid was used as a control and was kindly provided by Dr. Yuzhi Fu from the Wuhan Institute of Virology, Chinese Academy of Sciences. The PRDIII/I-luc and PRDII-Luc plasmids were purchased from Beyotime. pRL-TK (Promega Company, USA) expressing Renilla luciferase was used as endogenous transfection control to allow normalization.

\subsection{Cell Culture, Transfection, and Virus Infection}

Caprine EECs were kindly provided by Prof. Yaping Jin from the Northwest Agricultural University of China and were grown in Dulbecco's Modified Eagle Medium/F-12 (Thermo Fisher, Waltham, MA, USA) with 10\% fetal bovine serum (Gibco, Thermo Fisher). HEK-293T cells were grown in Dulbecco's Modified Eagle Medium with 10\% fetal bovine serum (Gibco, USA). Vero-SLAM cells expressing SLAM receptors were generated at the Shanghai Veterinary Research Institute and grown in Dulbecco's Modified Eagle Medium with $10 \%$ fetal bovine serum (Gibco, USA). Vero-SLAM cells support efficient replication of PPRV and the downstream signaling from caMAVS is functional. In a previous study, we demonstrated that nucleolin could inhibit PPRV replication in Vero-SLAMs [22]. All cells were grown with $1 \%$ antibiotics of penicillin and streptomycin $(\mathrm{P} / \mathrm{S})$ at $37^{\circ} \mathrm{C}$ and with a $5 \%$ atmospheric $\mathrm{CO}_{2}$ concentration. For transfection experiments, cells were seeded in 6-well plates (NEST Biotechnology, Jiangsu, China) overnight, and transfected with 
indicated expression plasmids and $36 \mathrm{~h}$ post transfection, cells were harvested and used for further experiments.

The PPRV vaccine strain, Nigeria/75/1 (GenBank: HQ197753), was obtained from the Shanghai Veterinary Research Institute cell culture collection and amplified on Vero-SLAM cells. After experiments were completed, all experimental materials were autoclaved at $120^{\circ} \mathrm{C}$ for $30 \mathrm{~min}$ to kill PPRV. The laboratory was confirmed to P2 (BSL-2) laboratory requirements and all experiments were completed under $\mathrm{P} 2$ laboratory conditions.

\subsection{Quantitative Real-Time PCR ( $q R T-P C R$ )}

qRT-PCR was performed to measure the mRNA levels of selected ISGs, including caprine RSAD2 (caRSAD2), caprine OASL (caOASL2), caprine IFITM3 (caIFITM3), caprine MX1 (caMX1). The caprine GAPDH was used as endogenous control and for normalization of the data. Gene-specific primers were designed by using Primer3 software or according to previous reports [23] and then synthesized by Sangon Biotech, China. Total RNA was extracted by using the TRIzol Reagent (Invitrogen), and cDNA synthesis was performed using M-MLV reverse transcriptase (Promega, USA) with Oligo dT primer or Random primers (Invitrogen). qRT-PCR was performed using SYBR Premix Ex Taq reagents (Takara, Dalian, China) and StepOne Real-Time PCR Detection System (Thermo Fisher, USA). Fold expression was presented to show the relative abundance of the mRNAs by using the comparative CT $(\Delta \Delta \mathrm{CT})$ method. All experiments were performed in triplicate and all experiments were carried out with at least three repeats.

\subsection{Reporter Plasmids and Luciferase Assays}

HEK-293T cells or EEC Cells $\left(1.25 \times 10^{5}\right)$ were cultured in 24-well plates. Transfections were performed with different amounts of expression plasmid for the full-length caMAVS to compare the activity of stimulating IFN- $\beta$-luc, and the same amount of full-length or mutant caMAVs to compare their difference in stimulating IFN- $\beta$-luc. Each caMAVS plasmid was co-transfected with the same amount of luciferase reporter plasmid and the pRL-TK plasmid, which is used for data normalization. Transfection with different amounts of expression plasmid for the full length caMAVS (250 ng, $500 \mathrm{ng}, 1 \mu \mathrm{g}$ ) was used to compare the induction of IFN- $\beta$-luc. The same amount of DNA was used to compare full-length caMAVS with the deletion mutants. The same amount of pRL-TK plasmid (40 ng/well) for data normalization was co-transfected along with $500 \mathrm{ng}$ of firefly luciferase reporter constructs (IFN- $\beta$-luc or PRD-III/I-luc and PRD-II-luc). Cells were collected at $24 \mathrm{~h}$ post-transfection. Luciferase activity was measured by using the DualLuciferase Reporter Assay kit (Promega, USA) according to the manufacturer's protocols. All reporter assays were repeated at least three times. The luciferase activity of each sample was normalized to the Renilla luciferase activity and all data were expressed as mean relative luciferase with standard deviation (SD). Furthermore, all experiments were performed at least three times independently.

\subsection{Confocal Immunofluorescence Microscopy}

To investigate the subcellular localization of the caprine MAVS protein, Vero-SLAM cells were plated on $15 \mathrm{~mm}$ cover glasses (NEST Biotechnology, China) and were transfected with Flag-tagged caMAVS (full-length or mutant versions) or together with the mitochondrial indicator plasmid pDsRed2-Mito (Addgene plasmid \# 55838) at 60 70\% confluence. Vero-SLAMs were selected for transfection instead of EECs because of the much higher transfection efficiency to achieve sufficient levels of simultaneous expression of two plasmids in one cell. The cells were harvested at $24 \mathrm{~h}$ post-transfection and fixed with $4 \%$ paraformaldehyde in PBS, and subsequently blocked with 5\% Bovine Serum Albumin (BSA) in PBS at $37^{\circ} \mathrm{C}$ for one hour. Immunofluorescence analysis was performed with primary antibodies (1:200, see next paragraph) directed against the tags and Alexa Fluor488-conjugated goat anti-mouse antibody (Invitrogen, USA) or Alexa Fluor-633-conjugated goat anti-rabbit antibody (Invitrogen, USA) (1:1000) as secondary antibodies, respectively. 
The cell nucleus was stained with DAPI (Beyotime, China) for 5 min and then washed four times with PBS-Tween. The images were taken with a Zeiss LSM880 confocal microscope and analysed by Zen Blue software (Zeiss, Germany).

\subsection{Immunofluorescence and Western Blot Analysis}

Anti-PPRV-N monoclonal antibody directed against the nucleocapsid protein $(\mathrm{N})$ of PPRV was generated by GenScript (Nanjing, China). Flag-tag primary antibodies were purchased from Sigma (USA). Myc-tag primary antibodies were purchased from Santa Cruz (USA), HA-tag primary antibodies were purchased from Abcam (UK). GAPDH and $\beta$-actin antibody were purchased from CoWin Biosciences (China). At 24-36 h after transfection with expression plasmids (caMAVS, huMAVS, or truncated caMAVS), cells were washed thoroughly with cooled PBS and lysed in cell lysis buffer containing a protease inhibitor cocktail (Merck Millipore, Darmstadt, Germany). The lysates were subjected to SDS-PAGE and transferred to nitrocellulose membranes (Whatman, Maidstone, UK). The membranes were then blocked at $37{ }^{\circ} \mathrm{C}$ with $5 \%$ skimmed milk for an hour and incubated with specific primary antibodies $(1: 1000)$ overnight at $4{ }^{\circ} \mathrm{C}$ and then subjected to horseradish peroxidase (HRP)-conjugated secondary antibodies (Jackson Immuno Research Laboratories) $(1: 10,000)$ for $1 \mathrm{~h}$ at room temperature. The bound secondary antibodies were visualized with enhanced chemiluminescence (ECL) (Thermo Fisher Scientific, Pittsburgh, PA, USA). For the virus infection experiment, cell lysates were harvested with indicated time intervals after infection and generated by adding $5 \times$ loading buffer to the collected cells and separated by SDS-PAGE and subjected to Western blot analysis. By using indicated primary antibodies which recognized different cellular proteins downstream of the PPRVinduced RIG-I pathway, including MAVS (Cell Signaling Technology, Danvers, MA, USA), TBK1 (CST, USA), P-TBK1 (CST, USA), the different protein expression levels were analyzed in the presence or absence of a PPRV infection. Fis-1 (CST, USA), which is a component of a mitochondrial complex, was used as indication for mitochondria.

\subsection{Co-Immunoprecipitation}

Co-immunoprecipitation (Co-IP) was performed to confirm the interaction between caRIG-I and caMAVS, and to determine whether PPRV protein V (PPRV-V) interacts with caMAVS. HEK-293T cells were seeded on $100 \mathrm{~mm}$ dishes and transfected with $5 \mu \mathrm{g}$ of the Myc-tagged caRIG-I encoding plasmid together with $5 \mu \mathrm{g}$ of HA-tagged wild-type (WT) or truncated caMAVS (caMAVS- $\triangle$ CARD, caMAVS- $\triangle \mathrm{PRR}$, caMAVS- $\triangle$ NC or caMAVS$\triangle \mathrm{TM}$ ) encoding plasmids. Alternatively, Flag-V was co-transfected together with MyccaMAVS. Then, $24 \mathrm{~h}$ post-transfection, cells were lysed with cell lysis buffer $(150 \mathrm{mM}$ $\mathrm{NaCl}, 50 \mathrm{mM}$ Tris- $\mathrm{HCl}, \mathrm{pH}$ 8.0, $5 \mathrm{mM}$ EDTA, 0.5\% NP-40) containing protease inhibitors (Merck-Millipore). Lysates were centrifuged at $12,000 \times \mathrm{g}$ for $10 \mathrm{~min}$ to remove the cell debris. Precipitation was performed overnight at $4{ }^{\circ} \mathrm{C}$ with anti-Myc mAb conjugated to Resin Agarose beads (Thermo Fisher Scientific). The beads were washed four times with lysis buffer and the bound proteins were eluted with SDS loading buffer by heating for $10 \mathrm{~min}$. Fifty microliter volumes of cell lysate were eluted and then subjected to Western blot analysis using specific antibodies.

\subsection{Statistical Analysis}

All statistical analysis was performed using GraphPad Prism Version 5. Data were expressed as means \pm standard deviations. A one-way ANOVA test was used to compare multiple groups $(>2)$. $p$ values $<0.05$ were considered to indicate significant differences.

\section{Results}

\subsection{Cloning and Sequence Analysis of Caprine MAVS Gene}

A 1566 bp-long caMAVS cDNA sequence was amplified by conventional RT-PCR. The nucleotide sequence of Capra hircus MAVS (caMAVS) has been submitted to GenBank (accession number: MT501722). The predicted 522 amino acid caMAVS protein shares the 
highest (98.1\%) amino acid similarity with the predicted sheep MAVS (XM_015099722.2). A neighbor-joining phylogenetic tree was constructed based on selected cDNA sequences of MAVS from several species (Figure 1A). The phylogenetic tree contains two major branches of mammalian MAVS, which are separated from avian and fish MAVS. In the MAVS amino acid sequence alignment, it was shown that caMAVS has the same structural domains as MAVS found in other mammalian species (Figure 1B).

\subsection{Schematic Representation and Identification of Caprine MAVS and Its Mutants}

Online structural analysis software (https: / / www.expasy.org/ (accessed on 1 December 2020)) predicts that caMAVS is a membrane-bound protein with a single C-terminal transmembrane (TM) domain. Based on the comparison with human MAVS and the predicted domains therein, in-frame deletion mutants of caprine MAVS were constructed to analyze the role of the CARD-domain, as well as the PRR, NC, and TM domains (Figure 2A). Western blotting was used to confirm the expression of caMAVS and its mutants before Luciferase and immunofluorescence assay (IFA) were conducted (Figure 2B).

\subsection{Subcellular Localization of Caprine MAVS}

Vero-SLAM cells were transfected with expression plasmids encoding caMAVS and mutants and subjected to IFA to determine the effect of the deletions on the subcellular location of caMAVS. As shown in Figure 3A, full-length caMAVS was abundantly present in the cytoplasm and had a punctate localization. The mutants caMAVS- $\triangle \mathrm{CARD}$ and cAMAVS- $\triangle$ PPR also had a punctate localization like the full-length caMAVS. However, when the TM or the NC domains were deleted, the mutant proteins lost their punctate localization and were dispersed over both the cytoplasm and the nucleus.

As the distribution of caMAVS to mitochondria is vital for its role in signaling, the subcellular location of caMAVS and the various mutants was compared with that of a co-transfected pDsRed2-Mito marker designed for fluorescently labeling mitochondria. As shown in Figure 3B, full-length MAVS co-localized with the mitochondrial marker, as well as caMAVS- $\triangle$ CARD and caMAVS- $\triangle P R R$. For caMAVS- $\triangle T M$ the mitochondrial localization was disrupted, suggesting that the mitochondrial colocalization was dependent on the TM domain. The caMAVS- $\triangle \mathrm{NC}$ mutant, from which a large (295 aa) central part of caMAVS was deleted, did also not colocalize with the mitochondrial marker.

\subsection{Overexpression of caMAVS-Induced IFN- $\beta$ via the NF- $\kappa B$ and IRF-3-Mediated Pathways}

As one of the most important adaptor molecules, MAVS is hypothesized to be critical for virus-induced type I IFN expression in goats, as a link between RLRs and downstream NF- $\kappa B$ and IRF3 activation [5]. To confirm that caMAVS is functional in the type I IFN pathway, HEK293T cells were co-transfected with plasmids expressing caMAVS and human IFN- $\beta$ promoter-driven luciferase. All cells were also co-transfected with pRL-TK encoding Renilla luciferase to allow for data normalization. As expected, overexpression of caMAVS activated the IFN- $\beta$ promoter in a dose-dependent manner (Figure 4A). However, the observed expression of luciferase was lower than with human MAVS, which acted as a positive control in this study. 
A

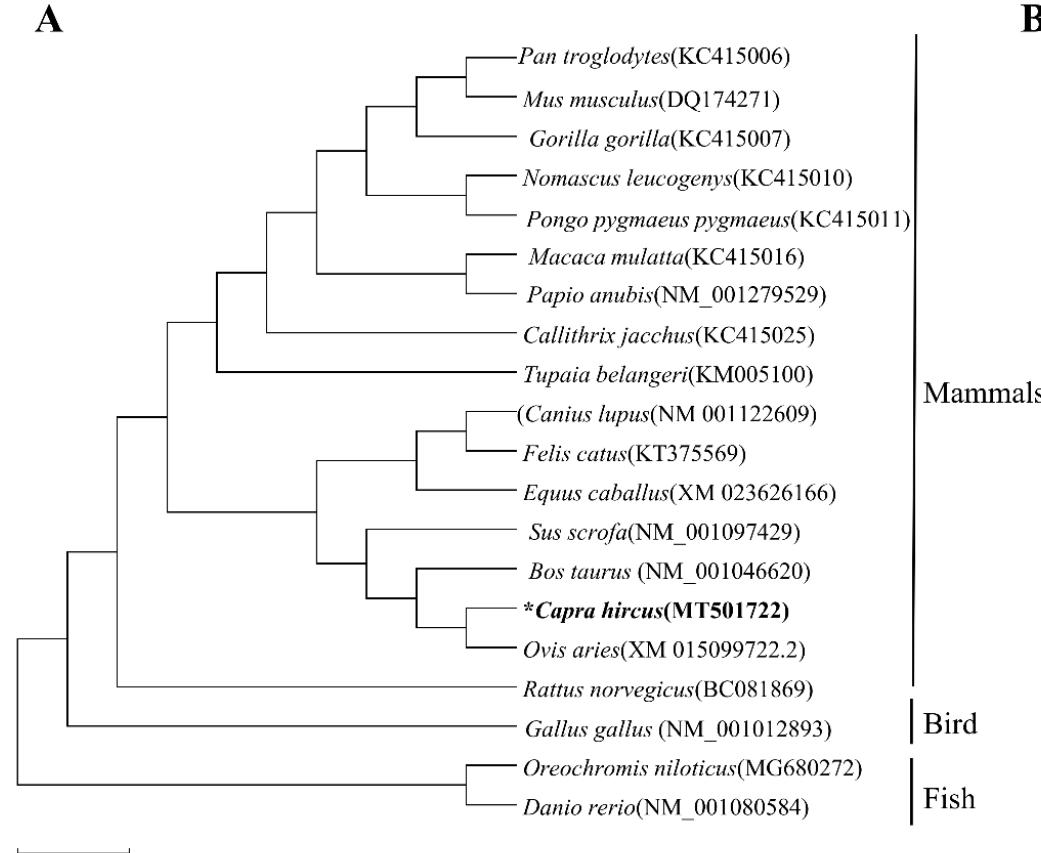

B

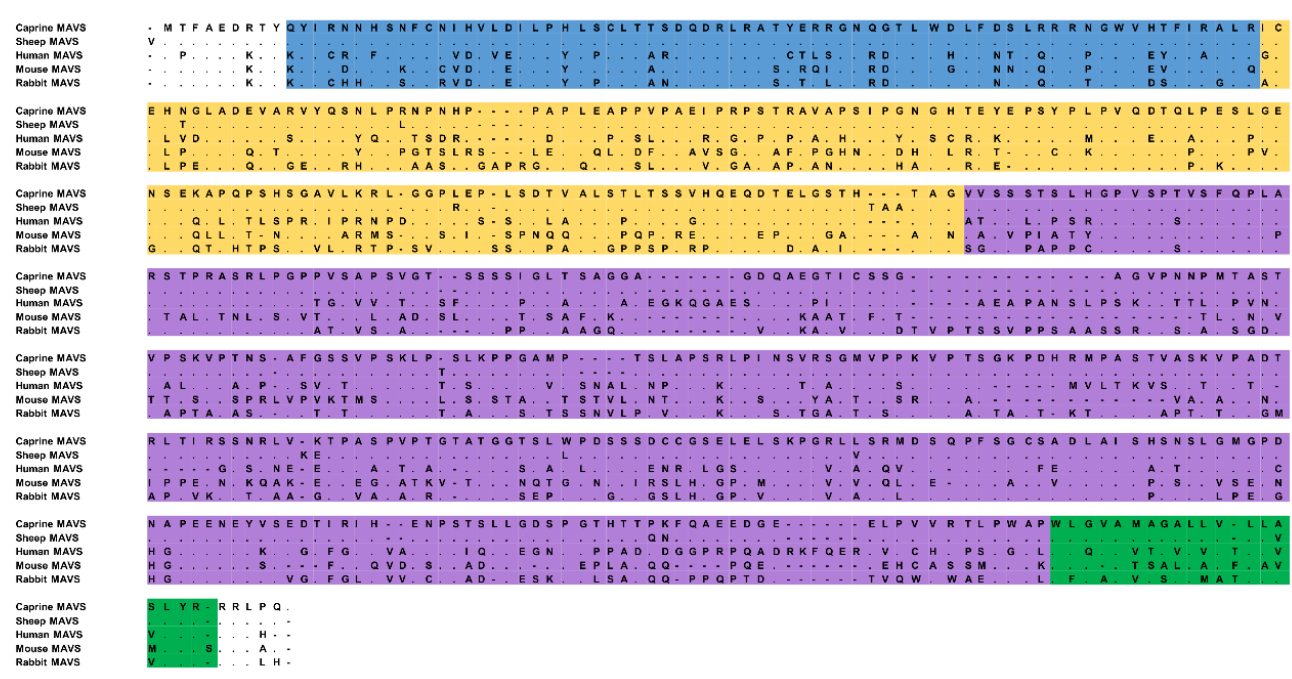

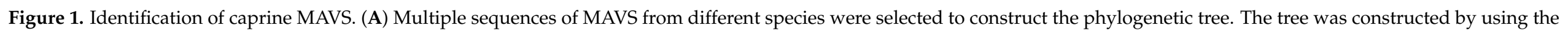

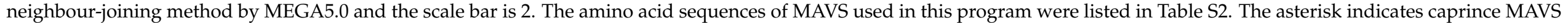

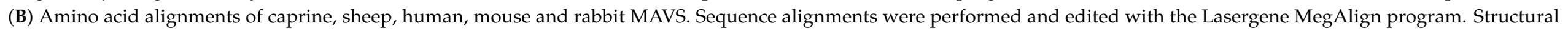

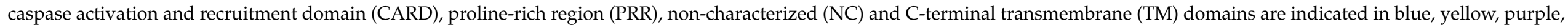
and green, respectively. 
A

HuMAVS

\begin{tabular}{|c|c|c|c|}
\hline CARD & Proline-rich & $\mathrm{NC}$ & TM \\
\hline
\end{tabular}

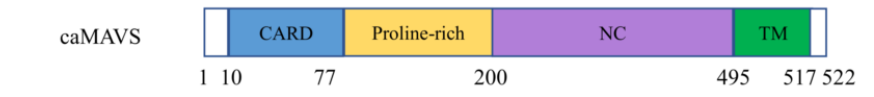

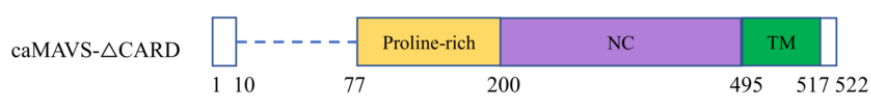

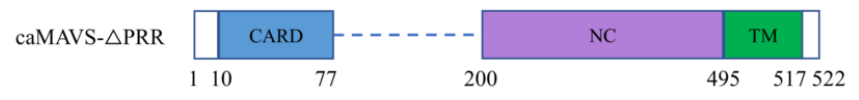

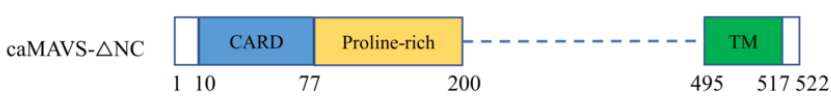

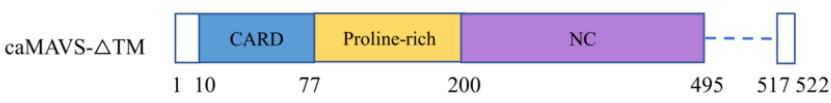

B

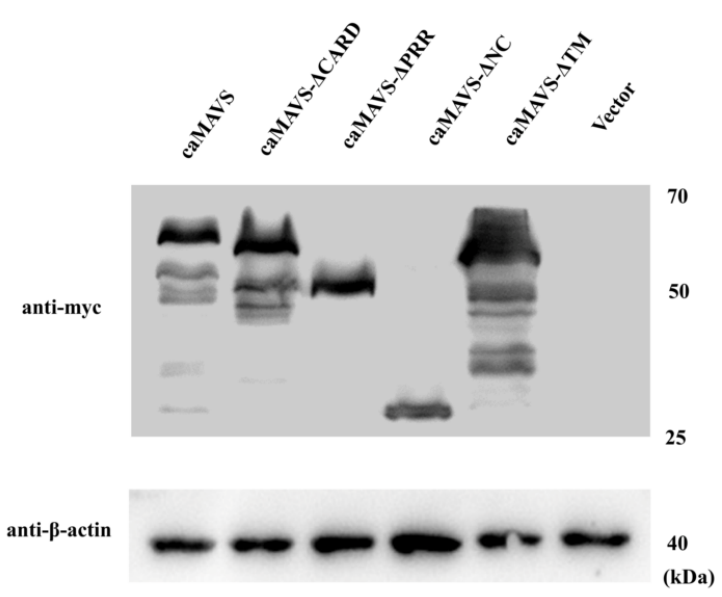

Figure 2. Schematic diagram of caprine MAVS mutants and their expression. (A) Schematic representation of caprine MAVS was presented based on the conserved points according to human MAVS. The CARD, PRR, NC and TM domains are represented in blue, yellow, purple, and green, respectively. The schematic representation is also used for constructing truncated mutants. The indicated numbers represent amino acid positions. (B) Western blot analysis of the expression of caMAVS and its mutants by transfection with caMAVS and mutant plasmids in HEK-293T cells.

A
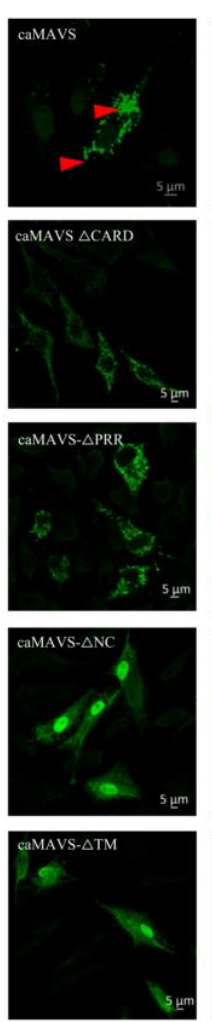
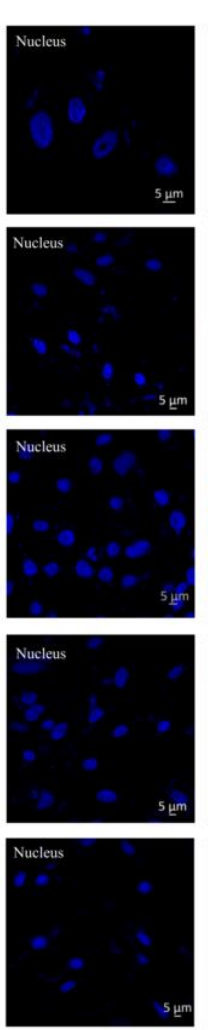
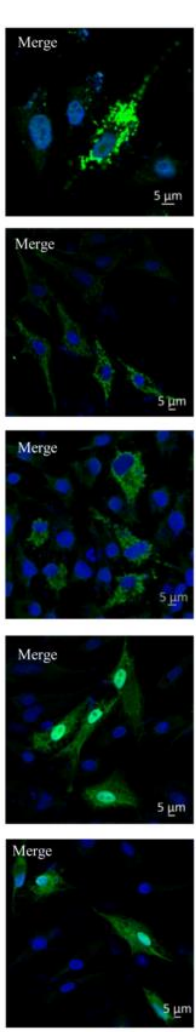

\section{B}
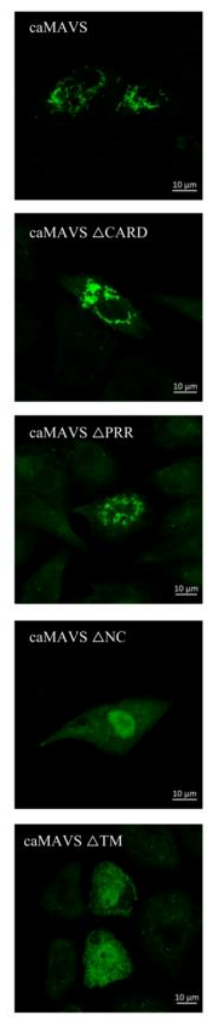
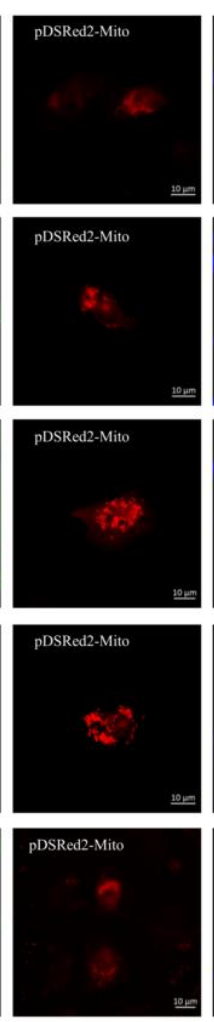
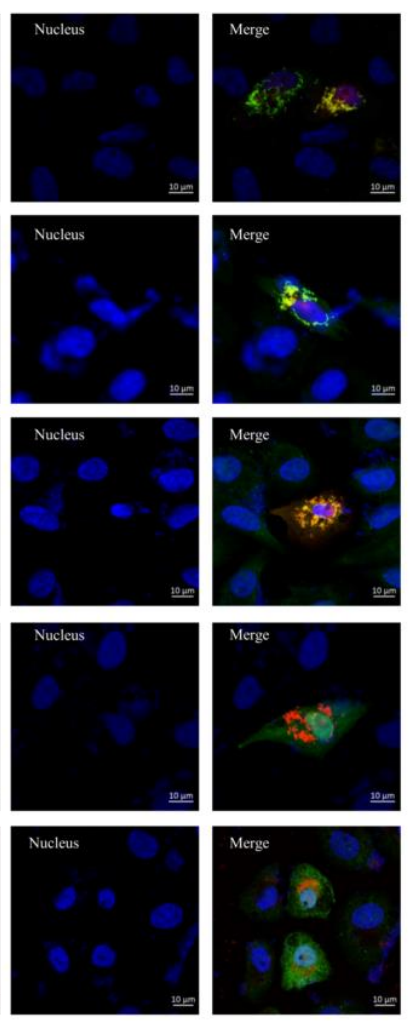

Figure 3. Localization study of caMAVS and its mutants. (A) Expression plasmids encoding caMAVS and its different deletion mutants were transfected into Vero-SLAM cells. At $36 \mathrm{~h}$ post transfection, cells were fixed and stained with Myc primary antibodies and secondary Alexa-488 labeled antibodies. (B) To verify the colocalization of caMAVS (green) and Mitochondria (red), expression plasmids encoding Myc tagged caMAVS and its mutants were transfected together with pDsRed2-Mit. At $36 \mathrm{hpt}$, cells were fixed and stained as in panel A. 
A

IFN- $\beta$-Luc

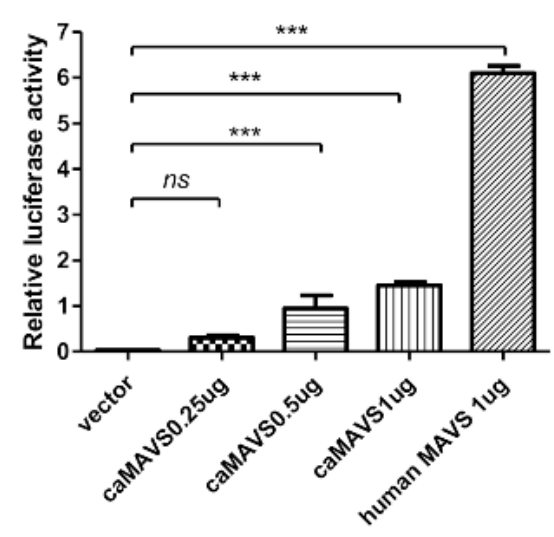

C

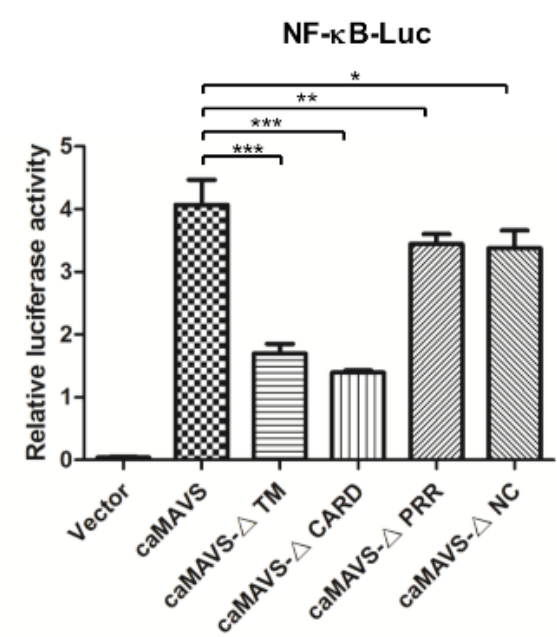

B

IFN- $\beta$-Luc

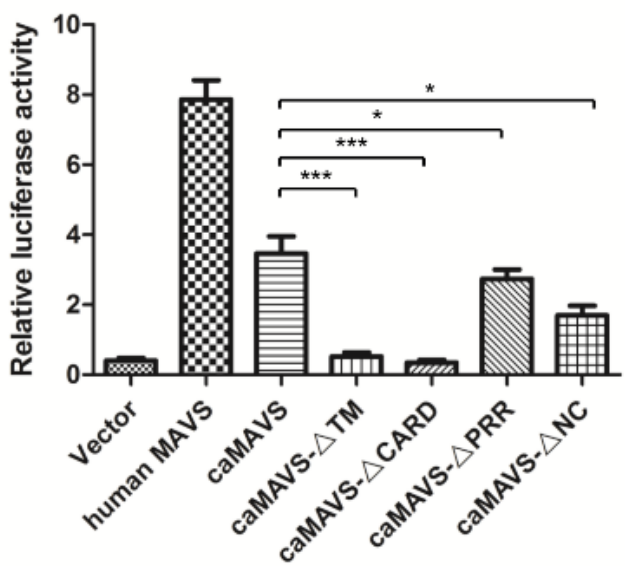

D

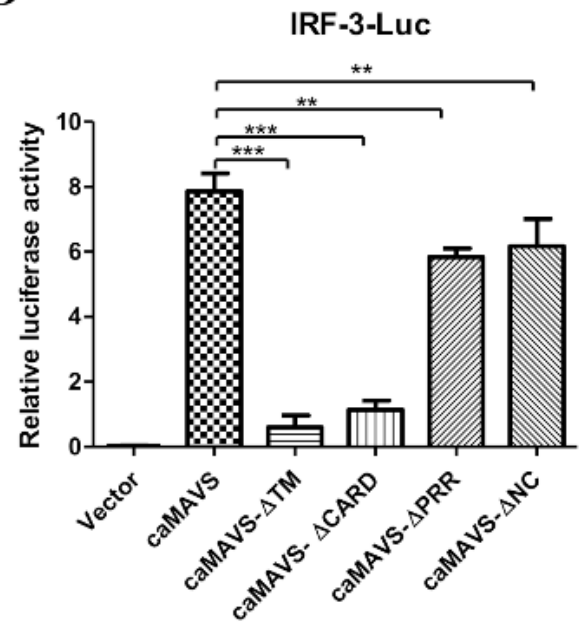

Figure 4. Overexpression of caMAVS induced IFN- $\beta$ via the NF- $\kappa B$ and IRF-3-mediated pathways. (A) HEK-293T cells were transfected with increasing amount of caMAVS, IFN- $\beta$-Luc together with endogenous control pRL-TK Plasmid (40 ng/well). (B-D) HEK-293T cells were transfected with caMAVS or its mutants (500 ng) along with PRDII-Luc (NK-kB-luc), PRDI/IIIluc (IRF3-luc)) or IFN $\beta$-Luc together endogenous control pRL-TK Plasmid (40 ng/well). Human MAVS was used as a positive control. At $36 \mathrm{hpt}$, the HEK-293T cells were lysed, and Rluc and Fluc activities were evaluated using the Promega Dual-Luciferase Reporter Assay System. Furthermore, all the experiments were performed at least three times to ensure the results consistency (The data represent the mean $\pm \mathrm{SD}$ of three independent experiments. One-way ANOVA was used for statistical analysis; ${ }^{*} p<0.05 ;{ }^{* *} p<0.01$; $\left.{ }^{* * *} p<0.001\right)$.

The next experiment illustrated that caMAVS mutants lacking either the CARD or the TM domains were unable to activate the IFN- $\beta$ promotor (Figure $4 \mathrm{~B}$ ), in line with previous analysis of mutants of feline MAVS [11]. In the next experiment, we used a set of luciferase report plasmids containing IRF-3 and NF-kB-binding motifs (PRDIII/I-Luc, referred to as IRF-3-Luc and PRDII-Luc, referred to as NF-kB-Luc) to further increase our understanding about caMAVS as an adaptor protein. HEK 293T cells were transfected with caMAVS, or mutant versions thereof, together with one of the luciferase reporter plasmids. The results demonstrated that overexpression of caMAVS, as well as the mutants caMAVS- $\triangle P R R$ and caMAVS- $\triangle N C$, could activate NF- $\kappa B$ and IRF-3 pathways, while caMAVS- $\triangle T M$ and caMAVS- $\triangle$ CARD had lost this ability (Figure $4 C, D)$. Together, the data demonstrated that caMAVS could activate the IFN- $\beta$ promoter through both NF- $\kappa B$ and IRF-3 signaling. 


\section{5. caMAVS Overexpression Upregulates the mRNA Level of Caprine ISGs}

IFN production results in the expression of IFN-stimulated genes (ISGs), which have an important role in morbillivirus antiviral innate immunity [23]. To further establish the role of caMAVS in ISGs induction, the effect of overexpression of caMAVS or its mutants on the transcription of a number of ISGs (caIFITM3, caOASL, caRSAD2, caMX1) was explored by qRT-PCR. Transfection of caMAVS in EECs resulted in the upregulation of the mRNA expression levels of all tested ISGs (Figure 5A). Furthermore, the deletion of either CARD or TM domains significantly limited the induction level of these ISGs (Figure 5B-E). All findings demonstrated that the CARD and TM domains are both essential for the MAVS-medicated IFN production.

A

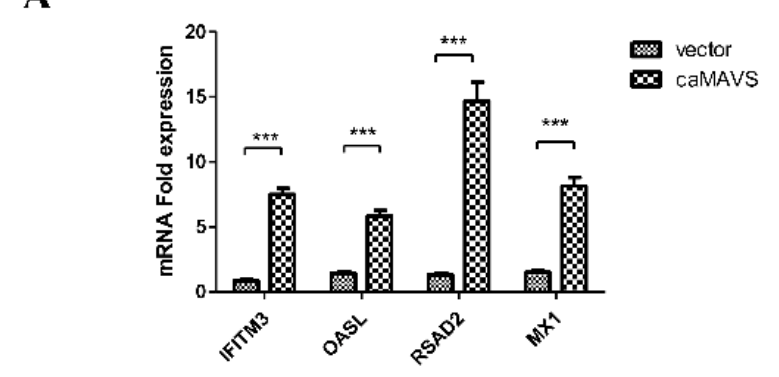

B
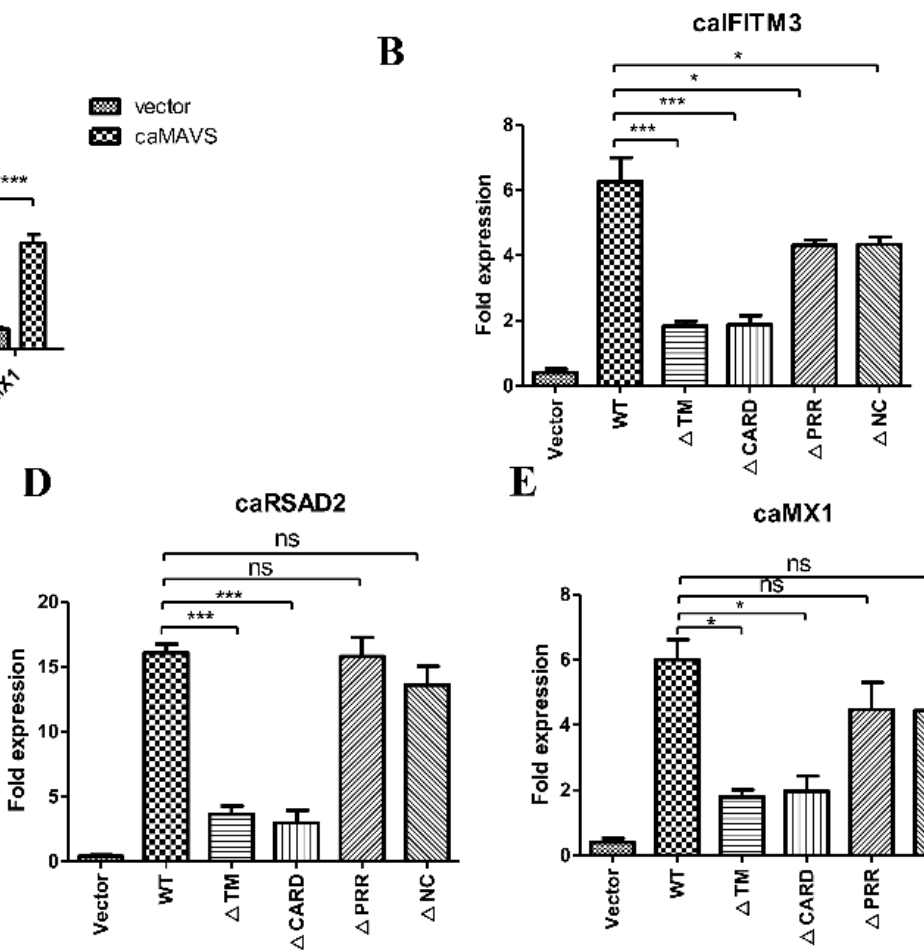

$\mathbf{E}$

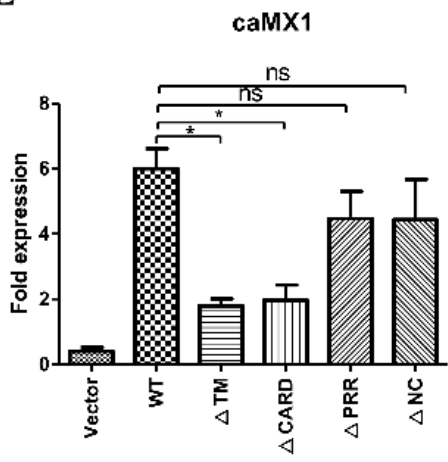

Figure 5. Evaluation of ISGs by overexpression of caMAVS and its mutants in EECs. (A) EECs were transfected with either empty vector or caMAVS. The relative mRNA levels of selected caprine ISGs (IFITM3, OASL, RASD2, MX1) were analyzed by qRT-PCR. (B-E) EECs were transfected with either empty vector or caMAVS and its mutants. The relative mRNA levels of selected ISGs were analyzed by qRT-PCR. Data presented were from at least three independent experiments. Significance was analyzed by GraphPad prism 5.0 software with a one-way ANOVA test (One-way ANOVA was used for statistical analysis; $\left.{ }^{*} p<0.05 ;{ }^{* *} p<0.01 ;{ }^{* * *} p<0.001\right)$.

\section{6. caMAVS Interacts with caRIG-I through Its CARD and NC Domain}

To verify that caMAVS functions as the adaptor of RIG-I, the caprine RIG-I gene was amplified and subcloned into pCMV-Myc (Clontech, USA) and tested in co-immunoprecipitation assays. HEK293T cells were transfected with HA-caMAVS (or its mutants), together with Myc-caRIG-I, and cell lysates were analyzed at $36 \mathrm{~h}$ post transfection. Myc-caRIG-I was precipitated with anti-Myc conjugated resin beads and Myc-caRIG-I and HA-caMAVS were detected using anti-bodies against the Myc and HA-tags, respectively. As shown in Figure 6A, the protein samples were incubated with Myc antibody and then the elution was subjected for HA antibody check. The results confirmed the interaction between caRIG-I and full-length caMAVS. Moreover, as shown in Figure 6B, the mutants with PPR or TM domains deleted still interacted with caRIG-I. On the other hand, the CARD domain deletion mutant displays a strongly reduced interaction with caRIG-I, indicating that the CARD domain of MAVS plays a key role in this interaction. Interestingly, also the NC 
domain mutant, which did not colocalize with mitochondria in the fluorescence studies also lost the ability to interact with caRIG-I.

A

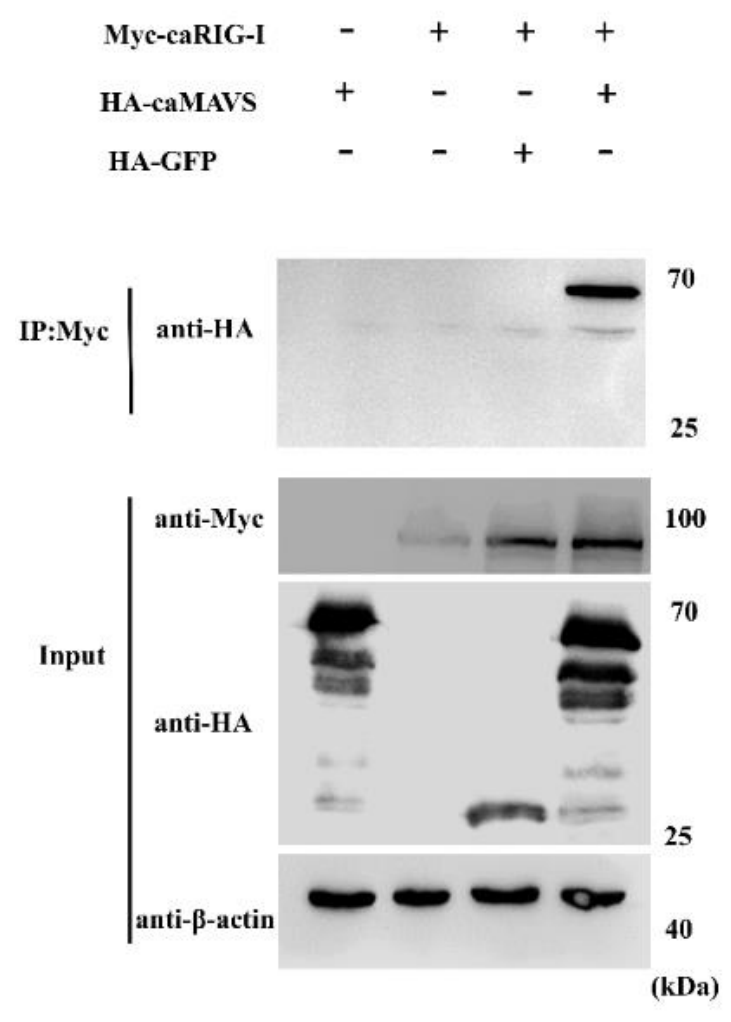

B
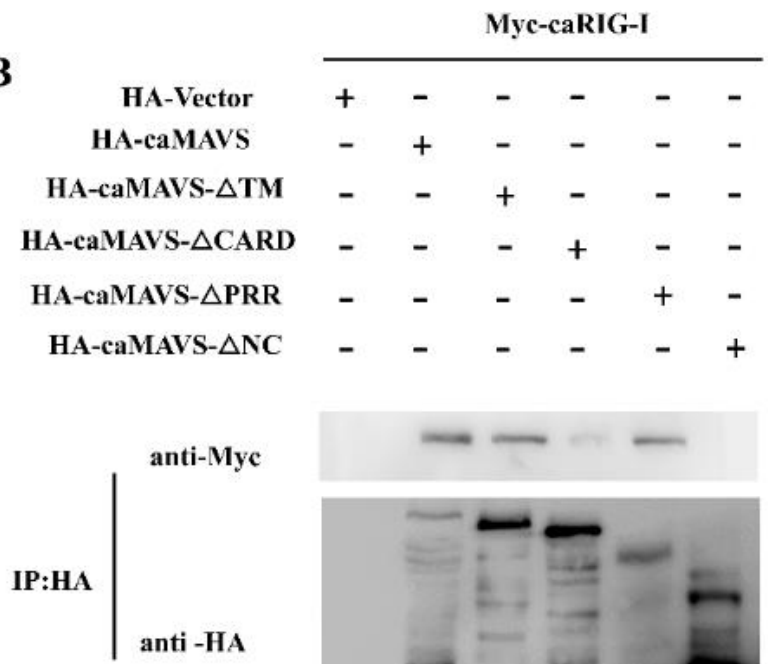

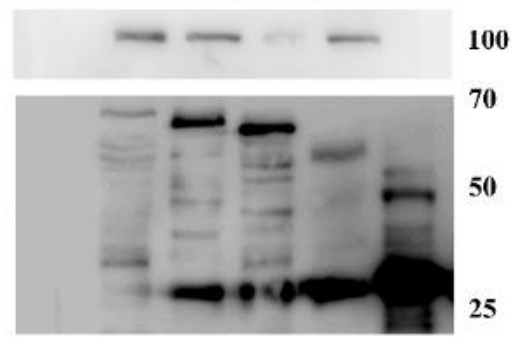

\section{0}

0

50 .

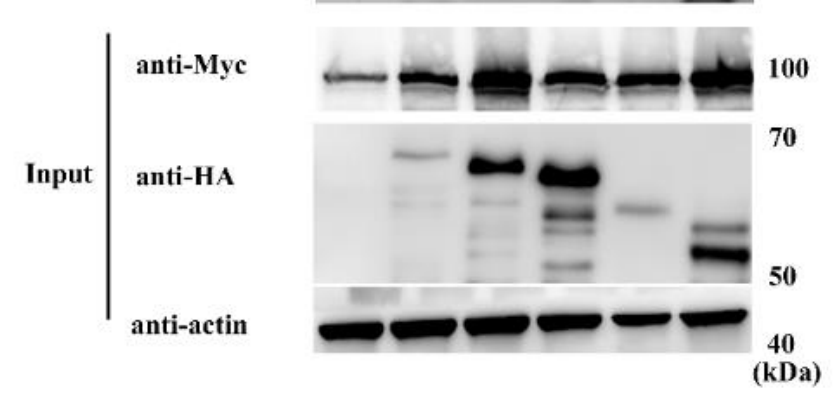

Figure 6. Identification of the interaction between caMAVS and caRIG-I by Co-IP. (A) HEK-293T cells seeded in $10 \mathrm{~cm}$ dishes and were transfected with HA-tagged MAVS or HA-GFP together with (without) Myc-RIG-I when cells were at around $60 \%$ confluency, respectively. Cells were harvested after $24 \mathrm{~h}$ post transfection. Cell lysates were precipitated with anti-Myc mAb resin overnight at $4{ }^{\circ} \mathrm{C}$ and precipitated proteins detected with anti-HA and anti-Myc mAbs. $\beta$-actin was used as protein loading control. (B) HEK-293T cells were seeded in $10 \mathrm{~cm}$ dishes and were transfected with HA-tagged MAVS and its mutants together with Myc-RIG-I when cells were around 60\% confluency. Cells were harvested after $24 \mathrm{hpt}$ and cell lysates were precipitated with anti-HA mAb resin overnight at $4{ }^{\circ} \mathrm{C}$ and precipitated proteins were detected with anti-HA and anti-Myc mAb. $\beta$-actin was used as protein loading control.

\section{7. caMAVS Response to PPRV Infection in EEC Cells}

PPRV has been responsible for severe infectious disease in small ruminants, including goats and sheep. The virus caused significant economic losses in the goat and sheep industry and also threatens wildlife conservation $[24,25]$. To determine where caMAVS functions as a key factor in the antiviral innate immune response to PPRV, EEC cells were infected with PPRV. Through screening for several proteins known to be important for the induction of innate immunity, we found that caMAVS was degraded after virus infection, as shown in Figure 7A. The endogenous protein expression level of the $70 \mathrm{kDa}$ MAVS was significantly decreased following infection with a high dose of PPRV. The $70 \mathrm{kDa}$ MAVS was downregulated, while the expression level of a smaller (45-50 kDa) band reactive with caMAVS antibodies was upregulated at $48 \mathrm{~h}$ with/without virus infection for reasons unknown. A truncated MAVS isoform was also identified in other species but was unable to trigger IFN production [26]. Furthermore, we observed that caMAVS mRNA levels remained relatively stable after PPRV infection (data not shown), which is consistent with other studies. In infections with other viruses, MAVS cleavage was a result of apoptosis-activated degradation [27], autophagic degradation [28] or ubiquitin- 
proteasome pathways [29]. Several inhibitors have been widely used to elucidate which pathway is involved in MAVS cleavage. MG132 is a commonly used proteasome inhibitor, chloroquine (CQ) is a classic inhibitor of autophagy, and Concanavalin A (ConA) induces autophagy. In our study, we found that the proteasome inhibitor MG132 was able to rescue caMAVS expression to mock-infection levels, in contrast to the lysosome inhibitor chloroquine (CQ). ConA treatment markedly reduced MAVS expression levels. Overall, this suggests that caMAVS degradation during PPRV infection is dependent on the ubiquitinproteasome pathway (Figure 7B).

A
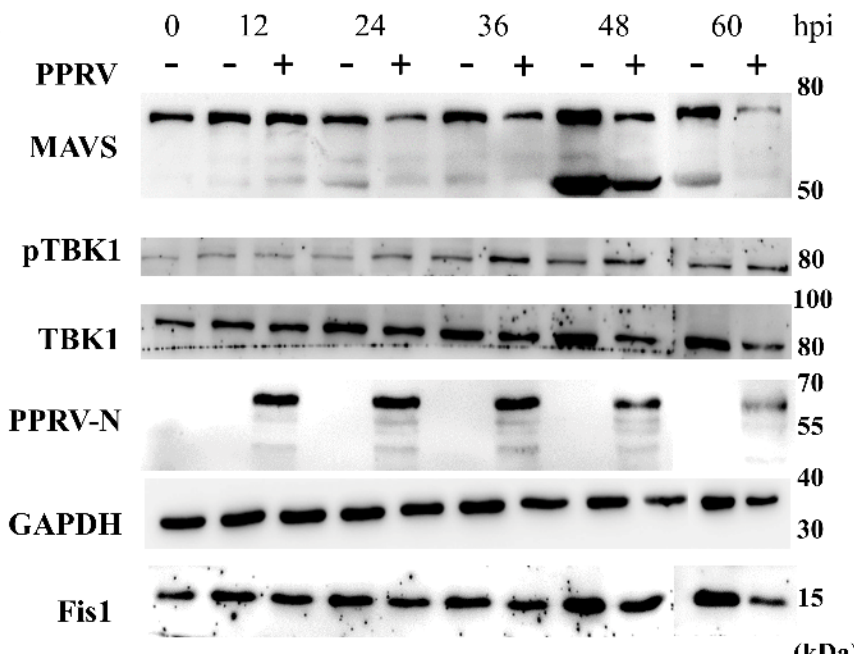

Gray level ratio

\begin{tabular}{l|l|l|l|l|l|l|l|l|l|l|l|} 
MAVS & 0.84 & 1.09 & 1.06 & 0.97 & 0.59 & 0.97 & 0.68 & 1.21 & 1.16 & 1.50 & 0.67 \\
\hline
\end{tabular}

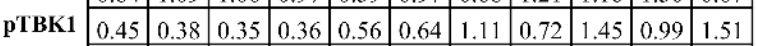

\begin{tabular}{|l|l|l|l|l|l|l|l|l|l|l|l|} 
TBK1 & 0.89 & 0.99 & 0.84 & 1.06 & 0.95 & 1.04 & 1.17 & 1.52 & 1.55 & 1.41 & 1.11 \\
\hline
\end{tabular}

\begin{tabular}{|l|lllllllll|l|l|l|}
\hline PPRV-N & 0.08 & 0.04 & 0.99 & 0.06 & 1.10 & 0.03 & 1.09 & 0.05 & 1.22 & 0.05 & 0.89 \\
\cline { 2 - 10 }
\end{tabular}

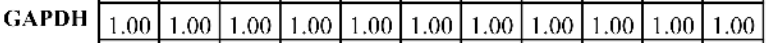

\begin{tabular}{l|l|l|l|l|l|l|l|l|l|l|l|} 
Fis1 1 & 0.79 & 1.06 & 0.82 & 0.97 & 0.95 & 1.02 & 1.02 & 1.24 & 1.57 & 1.66 & 1.05 \\
\hline
\end{tabular}

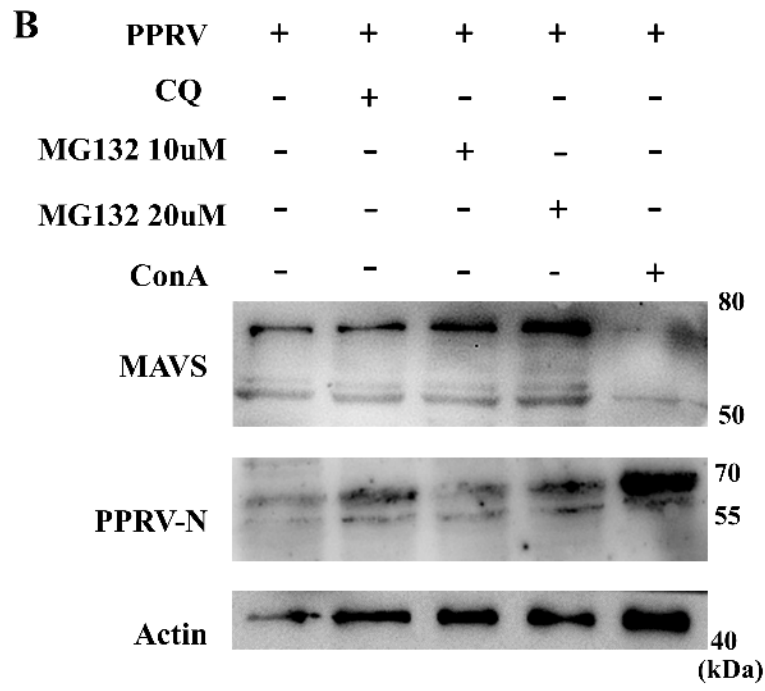

Gray level ratio

\begin{tabular}{r|c|c|c|c|c|} 
MAVS & 0.70 & 0.77 & 1.55 & 1.92 & 0.18 \\
\cline { 2 - 6 } PPRV-N & 0.89 & 0.74 & 0.68 & 1.18 & 1.20 \\
\cline { 2 - 6 } Actin & 1.00 & 1.00 & 1.00 & 1.00 & 1.00 \\
\cline { 2 - 6 } & \multicolumn{3}{|c}{} & \multicolumn{3}{|c}{} & &
\end{tabular}

Figure 7. caMAVS is degraded through the proteasome pathway during PPRV infection. (A) EECs were infected with the PPRV vaccine strain, Nigeria/75/1, at MOI 10. The relative protein level of several important molecules during viral infection was checked by WB. (B) Several inhibitors were used after infection of PPRV on EECs to demonstrate which pathway is involved in MAVS degradation. The relative level of individual proteins was quantified with respect to $\beta$-actin.

\subsection{PPRV V Protein Interacted with caMAVS}

To identify a putative viral protein responsible for caMAVS degradation in PPRVinfected cells, HEK-293T cells were co-transfected with viral protein-encoding plasmids $\mathrm{V}$ and Myc-caMAVS because Newcastle Disease Virus (NDV) V could target MAVS degradation to inhibit host type I interferon production [30]. Clearly, caMAVS expression level was greatly reduced during co-transfection with PPRV-V (Figure 8A). Co-IP assays between with the flagged tagged V protein of PPRV and Myc-caMAVS demonstrated that PPRV-V and caMAVs co-immunoprecipitated, irrespective of which of the two proteins was targeted for pull-down, indicative of a (direct) interaction between these two proteins (Figure 8B). Furthermore, Flag-V co-localized with Myc-caMAVS and with the mitochondrial marker pDsRed2-Mito (Figure 8C) when overexpressed in Vero-SLAM cells, indicating that PPRV-V most likely acts on mitochondrial MAVS. 
A

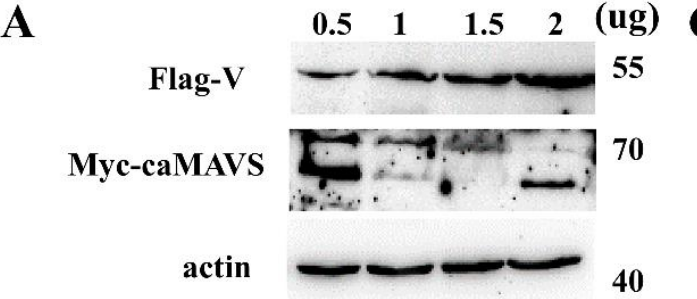

B

actin

40
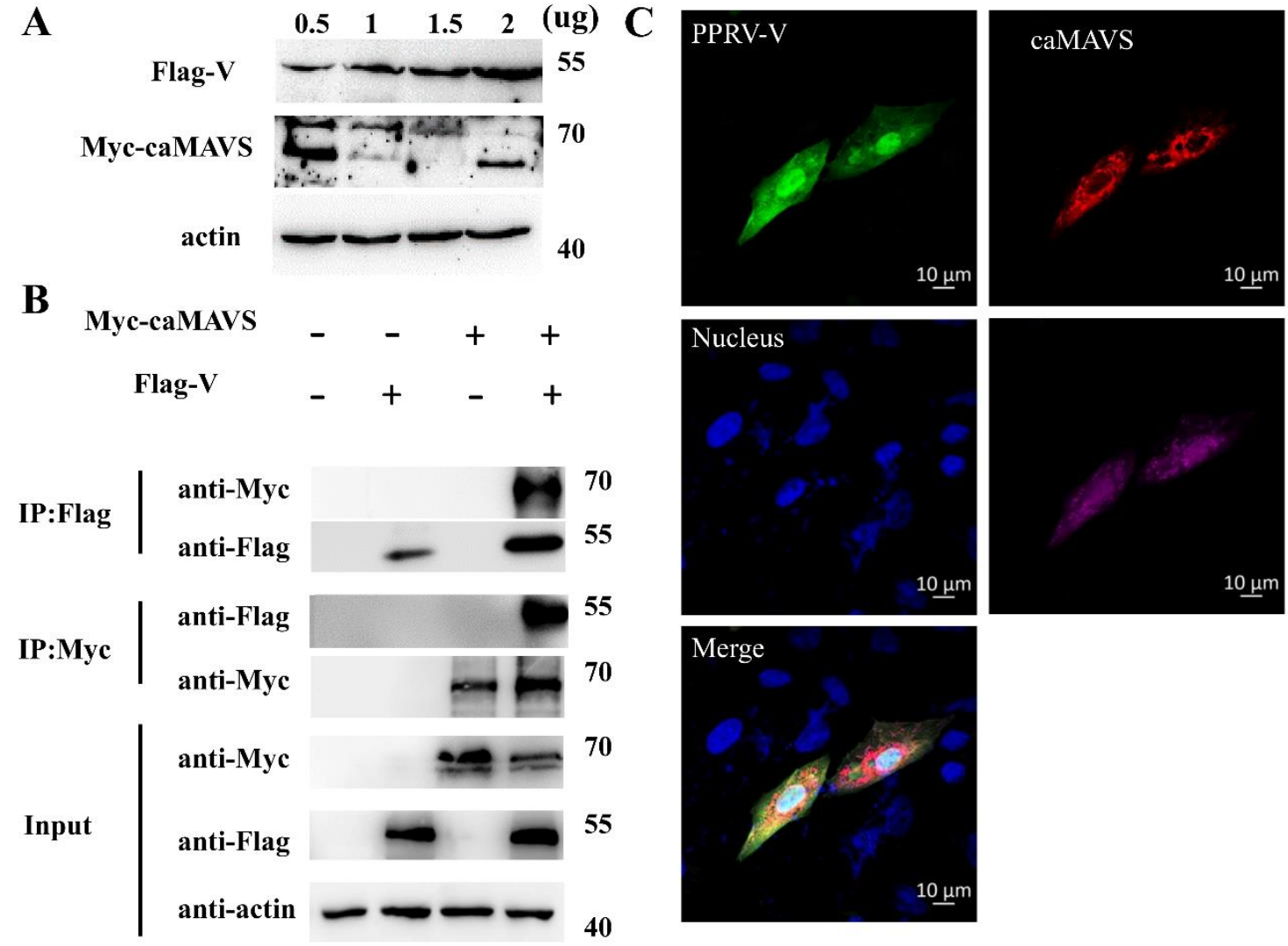

(kDa)

Figure 8. caMAVS interacts and colocalizes with PPRV viral protein V. (A) Increasing amounts of plasmids encoding Flagtagged PPEV protein V (Flag-V) $(0.5 \mathrm{ug}, 1 \mathrm{ug}, 1.5 \mathrm{ug}, 2 \mathrm{ug})$ were co-transfected with plasmid-encoding Myc-tagged caMAVS ( $2 \mathrm{ug}$ ) in 6-well plates. PPRV V and caMAVS were detected by Western blot using $\beta$-actin as loading control. (B) Co-IP was performed to identify interaction between PPRV V and caMAVS. Flag-V and Myc-caMAVS plasmids were transfected into HEK-293T cells independently or together. Cell lysates were subjected to immunoprecipitation with anti-Myc or anti-Flag mAb overnight at $4{ }^{\circ} \mathrm{C}$. The expression of the transfected proteins was determined by Western blotting by anti-Flag and anti-Myc mAbs, respectively. (C) The colocalization study was performed by transfection of Myc-caMAVS (red), Flag-V (green) together with pDsRed2-Mito (pink) and subjected for IFA with anti-Flag and anti-Myc mAbs as primary antibodies.

\section{Discussion}

PPRV is the causative agent of a highly contagious disease that affects both domestic and wild small ruminants [24]. Except for farm animals, studies also reported the infection of PPRV lineage II in the Chinese water deer, Hydropotes inermis [31] and recent outbreaks of PPRV have also been reported to occur in other wild animals in different countries [32,33]. PPRV appears to induce immune suppression during the acute phase of the disease [34], which may favor the establishment of secondary infections with other pathogens. Recently, it was demonstrated that PPRV utilizes the viral Nucleoprotein $(\mathrm{N})$ and Phosphoprotein (P) to inhibit interferon signalling by blocking the JAK-STAT Pathway [35]. Another study showed that the N protein inhibits IFN- $\beta$ production by interacting with IRF3 [36]; however, up to now, studies on PPRV were mostly performed in the human cell line 293T, while humans are no natural PPRV host.

We hypothesized that PPRV may also inhibit other immune signaling pathways to effectively evade innate immune responses. Interference with the RIG-I-like receptor (RLR) mediated pathways, for instance, might lead to a reduction in the production of ISGs. The activity of this pathway during a PPRV infection was studied by analysing the MAVS adaptor protein in caprine cells. MAVS is crucial in the RIG-I/MDA-5 sensing of various RNA viruses and has been characterized in several species as a key factor in 
RLR pathways. In our study, we successfully cloned the MAVS homolog from caprine endometrial epithelial cells (EECs), a host cell line for PPRV. By sequence comparison, we found that caMAVS shares conserved structural domains (CARD-PRR-NC-TM) with MAVS of other mammalian species and that caMAVS shares the highest identity with sheep MAVS (98.1\%). Further analysis using transiently expressed MAVS mutants mapped individual domains of caMAVS responsible for its distribution in Vero-SLAM cells, which have been described as a useful cell model to study PPRV infection [22]. The full-length caMAVS protein was localized in mitochondria, while caMAVS lacking the TM domain lost the mitochondrial localization and was found in both cytoplasm and nucleus. Further, by using a luciferase-based assay, caMAVS lacking the deleted CARD domain did not induce IFN- $\beta$ reporter activity and did not activate downstream ISGs. Interestingly, caMAVS carrying a deletion of the NC domain, which has not been studied by others before, also lost mitochondrial localization, but could still induce IFN- $\beta$-Luc, NF-kB-Luc, and IRF-3-Luc, albeit less efficiently than the full-length caMAVS. A potential limitation of the luciferase-based assay is the use of HEK 293T cells instead of a natural host cell line (e.g., EECs), but the very low transfection efficiency in such cells precluded us from generating meaningful results. Given that PPRV can replicate in primate cells, the transfection efficiency or reporter plasmids in HEK293T cells is much higher, and the fact that caMAVS overexpression induces IFN- $\beta$ transcription, we considered this a suitable alternative cell model. Because the induction of downstream signalling by overexpressed caMAVS is independent from interaction with RIG-I, this may explain why deletion of the NC domain abrogates interaction with RIG-I (Figure 6B) but can still induce downstream signalling (Figures 4 and 5). Similarly, in a mouse model of hantaan orthohantavirus (HTNV, genus Orthohantavirus, family Hantaviridae), it has been observed that IFN production is MAVS independent [37].

By activation of innate immunity, host cells aim to restrict the spread of invading viruses and other pathogens. Once the infection is cleared, cells have their own system to downregulate such responses to return to a normal physiological state. The cellular proteins PCBP2 [38] and PCBP1 [39] can mediate the degradation of the adaptor protein MAVS. NLRX1 (a nucleotide binding oligomerization domain (NOD)-like receptor X1) recruits PCBP2 to induce MAVS degradation through the proteasomal pathway [40]. During $\mathrm{HCV}$ infection, the cellular Golgi protein 73 (GP73) acts as a negative regulator of innate immunity by interacting with MAVS/TRAF6 to promote degradation of this complex [41]. Alternatively, viruses may also encode proteins to specifically downregulate MAVS. For example, MAVS is degraded by the Rotavirus (RV) RNA methyl- and guanylyl-transferase (VP3) in a host-range-restricted manner [42]. Likewise, MAVS is cleaved followed by infection with the Avian infectious bronchitis virus [43]. In our study, we observed that PPRV infection induced MAVS degradation in host cells. Moreover, by testing a series of inhibitors, we found that PPRV-induced caMAVS degradation in EECs was medicated by the proteasome. When EECs were infected with a high MOI, the degradation of caMAVS was significant within $36 \mathrm{~h}$ post-infection. At the same time, the expression level of a smaller (45-50 $\mathrm{kDa}$ ) polypeptide that also reacted with the caMAVS antibodies increased from $36 \mathrm{~h}$ post infection (Figure 7A), suggesting that caMAVS variants might exist, that may possess diverse biological functions in innate immune regulation. Surprisingly, we also found that the truncated variant is also significantly visible at the $48 \mathrm{~h}$ point without virus infection; however, it also decreased later at the $60 \mathrm{~h}$ point, which remains unclear. Truncated variants of MAVS (mini MAVS), which is expressed from a bicistronic mRNA of MAVS have been demonstrated to possess the ability to antagonize the signalling function of MAVS and thereby downregulate type I IFN expression by regulation of cell death as described previously [26].

Finally, we showed that overexpression of the PPRV V protein, in absence of a virus infection, also led to the downregulation of caMAVS. Although we did observe co-localization of PPRV-V with caMAVS in transfected cells, further studies will be required to fully understand the degradative interplay between PPRV-V and caMAVS. Interestingly, PPRV-C 
protein has recently been demonstrated to inhibit IFN- $\beta$ induction by interacting with RIG-I and MAVS [44], which might indicate that both PPRV-V and PPRV-C are critical for counteracting the innate immune response. Since the results described in this study are based on the PPRV vaccine strain from lineage II, it would be interesting to follow up on this research to investigate commonalities and potential differences between the four circulating lineages [45] in their ability to counteract caMAVS.

In summary, our study characterized a MAVS homolog from immortalized goat cells (EECs) and investigated the functions of caMAVS during type I IFN signalling. We identified caMAVS as a RIG-I interacting type I interferon inducer and showed that caMAVS degraded during PPRV infection, possibly due to an interaction of PPRV-V and caMAVS.

Supplementary Materials: The following are available online at https:/ / www.mdpi.com/1999-491 5/13/3/409/s1, Table S1: Primers designed and used in this study; Table S2: The sequence of MAVS selected and included for polygenetic tree construction.

Author Contributions: Conceptualization, Q.M., G.L., G.P.P.; methodology, Q.M., R.Q., C.M., D.D.; experimental work Q.M., R.Q.; data analysis, Q.M., R.Q., J.Z., A.T., D.D., H.G.; writing—original draft preparation, Q.M.; writing—review and editing, G.L., M.M.v.O., G.P.P.; supervision, M.M.v.O., G.L., G.P.P.; funding acquisition, M.M.v.O., G.L., J.Z. All authors have read and agreed to the published version of the manuscript.

Funding: This study was supported by the National Key Research and Development Program of China (No. 2016YFD0500108), the National Natural Science Foundation of China (No. 31672572), Shanghai Sailing program (20YF1457700), the China postdoctoral Science Foundation (2019M660885), the State Key Laboratory of Genetically Engineered Veterinary Vaccines (No. AGVSKL-ZD-202010), the Fundamental Research Funds for the Central Institutes Program (2020JB06) and the Science and Technology Promoting Agriculture Innovation Project of Shanghai (No. 2019 No.3-3). Qiuhong Miao is enrolled through the graduate school Production Ecology and Resource Conservation (PE\&RC) in the 2016 Sino-Dutch joint Ph.D. program between the Chinese Academy of Agricultural Sciences (CAAS) and Wageningen University \& Research. The funders had no role in the study design, data collection, interpretation, or the decision to submit the work for publication.

Data Availability Statement: Data is contained within the article or supplementary material.

Acknowledgments: We thank Zhidong Zhang and Yuzhi Fu for insightful suggestions.

Conflicts of Interest: The authors declare that there are no conflict of interest.

\section{References}

1. Kawai, T.; Akira, S. The roles of TLRs, RLRs and NLRs in pathogen recognition. Int. Immunol. 2009, 21, 317-337. [CrossRef] [PubMed]

2. Takeuchi, O.; Akira, S. Innate immunity to virus infection. Immunol. Rev. 2009, 227, 75-86. [CrossRef]

3. Wilkins, C.; Gale, M. Recognition of viruses by cytoplasmic sensors. Curr. Opin. Immunol. 2010, 22, 41-47. [CrossRef]

4. Yoneyama, M.; Fujita, T. Recognition of viral nucleic acids in innate immunity. Rev. Med. Virol. 2010, 20, 4-22. [CrossRef]

5. Seth, R.B.; Sun, L.; Ea, C.-K.; Chen, Z.J. Identification and Characterization of MAVS, a Mitochondrial Antiviral Signaling Protein that Activates NF-kB and IRF3. Cell 2005, 122, 669-682. [CrossRef]

6. Xu, L.-G.; Wang, Y.-Y.; Han, K.-J.; Li, L.-Y.; Zhai, Z.; Shu, H.-B. VISA Is an Adapter Protein Required for Virus-Triggered IFN- $\beta$ Signaling. Mol. Cell 2005, 19, 727-740. [CrossRef]

7. Kawai, T.; Takahashi, K.; Sato, S.; Coban, C.; Kumar, H.; Kato, H.; Ishii, K.J.; Takeuchi, O.; Akira, S. IPS-1, an adaptor triggering RIG-I- and Mda5-mediated type I interferon induction. Nat. Immunol. 2005, 6, 981-988. [CrossRef]

8. Meylan, E.; Curran, J.; Hofmann, K.; Moradpour, D.; Binder, M.; Bartenschlager, R.; Tschopp, J. Cardif is an adaptor protein in the RIG-I antiviral pathway and is targeted by hepatitis C virus. Nature 2005, 437, 1167-1172. [CrossRef]

9. Liu, Y.; Lu, N.; Yuan, B.; Weng, L.; Wang, F.; Liu, Y.J.; Zhang, Z. The interaction between the helicase DHX33 and IPS-1 as a novel pathway to sense double-stranded RNA and RNA viruses in myeloid dendritic cells. Cell. Mol. Immunol. 2014, 11, 49-57. [CrossRef]

10. Xu, L.; Yu, D.; Peng, L.; Fan, Y.; Chen, J.; Zheng, Y.-T.; Wang, C.; Yao, Y.-G. Characterization of a MAVS ortholog from the Chinese tree shrew (Tupaia belangeri chinensis). Dev. Comp. Immunol. 2015, 52, 58-68. [CrossRef]

11. Wu, H.; Zhang, X.; Liu, C.; Liu, D.; Liu, J.; Wang, G.; Tian, J.; Qu, L. Molecular cloning and functional characterization of feline MAVS. Immunol. Res. 2016, 64, 82-92. [CrossRef] 
12. Sun, Y.; Mao, X.; Zheng, H.; Wu, W.; Rehman, Z.U.; Liao, Y.; Meng, C.; Qiu, X.; Tan, L.; Song, C.; et al. Goose MAVS functions in RIG-I-mediated IFN- $\beta$ signaling activation. Dev. Comp. Immunol. 2019, 93, 58-65. [CrossRef]

13. Huang, B.; Zhang, L.; Du, Y.; Xu, F.; Li, L.; Zhang, G. Characterization of the Mollusc RIG-I/MAVS Pathway Reveals an Archaic Antiviral Signalling Framework in Invertebrates. Sci. Rep. 2017, 7, 8217. [CrossRef]

14. Deng, J.; Chen, Y.; Liu, G.; Ren, J.; Go, C.; Ivanciuc, T.; Deepthi, K.; Casola, A.; Garofalo, R.P.; Bao, X. Mitochondrial antiviral-signalling protein plays an essential role in host immunity against human metapneumovirus. J. Gen. Virol. 2015, 96, 2104-2113. [CrossRef]

15. Sun, Q.; Sun, L.; Liu, H.-H.; Chen, X.; Seth, R.B.; Forman, J.; Chen, Z.J. The Specific and Essential Role of MAVS in Antiviral Innate Immune Responses. Immunity 2006, 24, 633-642. [CrossRef]

16. Dixit, E.; Boulant, S.; Zhang, Y.; Lee, A.S.Y.; Odendall, C.; Shum, B.; Hacohen, N.; Chen, Z.J.; Whelan, S.P.; Fransen, M.; et al. Peroxisomes Are Signaling Platforms for Antiviral Innate Immunity. Cell 2010, 141, 668-681. [CrossRef] [PubMed]

17. Horner, S.M.; Liu, H.M.; Park, H.S.; Briley, J.; Gale, M. Mitochondrial-associated endoplasmic reticulum membranes (MAM) form innate immune synapses and are targeted by hepatitis C virus. Proc. Natl. Acad. Sci. USA 2011, 108, 14590-14595. [CrossRef]

18. Weinberg, S.E.; Sena, L.A.; Chandel, N.S. Mitochondria in the regulation of innate and adaptive immunity. Immunity 2015, 42, 406-417. [CrossRef] [PubMed]

19. Rojas, J.; Sevilla, N.; Martín, V. PPRV-Induced Immunosuppression at the Interface of Virus-Host Interaction. Br. J. Virol. 2016, 3, 140-160. [CrossRef]

20. Sanz Bernardo, B.; Goodbourn, S.; Baron, M.D. Control of the induction of type I interferon by Peste des petits ruminants virus. PLOS ONE 2017, 12, e0177300.

21. Zhu, J.; Miao, Q.; Tang, J.; Wang, X.; Dong, D.; Liu, T.; Qi, R.; Yang, Z.; Liu, G. Nucleolin mediates the internalization of rabbit hemorrhagic disease virus through clathrin-dependent endocytosis. PLoS Pathog. 2018, 14, e1007383. [CrossRef]

22. Dong, D.; Zhu, S.; Miao, Q.; Zhu, J.; Tang, A.; Qi, R.; Liu, T.; Yin, D.; Liu, G. Nucleolin (NCL) inhibits the growth of peste des petits ruminants virus. J. Gen. Virol. 2020, 101, 33-43. [CrossRef]

23. Wani, S.A.; Sahu, A.R.; Khan, R.I.N.; Pandey, A.; Saxena, S.; Hosamani, N.; Malla, W.A.; Chaudhary, D.; Kanchan, S.; Sah, V.; et al. Contrasting Gene Expression Profiles of Monocytes and Lymphocytes From Peste-Des-Petits-Ruminants Virus Infected Goats. Front. Immunol. 2019, 10, 1463. [CrossRef]

24. Baron, M.D.; Diallo, A.; Lancelot, R.; Libeau, G. Peste des Petits Ruminants Virus. Adv. Virus Res. 2016, 95, 1-42.

25. Dou, Y.; Liang, Z.; Prajapati, M.; Zhang, R.; Li, Y.; Zhang, Z. Expanding Diversity of Susceptible Hosts in Peste Des Petits Ruminants Virus Infection and Its Potential Mechanism Beyond. Front. Vet. Sci. 2020, 7, 66. [CrossRef]

26. Brubaker, S.W.; Gauthier, A.E.; Mills, E.W.; Ingolia, N.T.; Kagan, J.C. A bicistronic MAVS transcript highlights a class of truncated variants in antiviral immunity. Cell 2014, 156, 800-811. [CrossRef]

27. Scott, I.; Norris, K.L. The mitochondrial antiviral signaling protein, MAVS, is cleaved during apoptosis. Biochem. Biophys. Res. Commun. 2008, 375, 101-106. [CrossRef]

28. Jin, S.; Tian, S.; Luo, M.; Xie, W.; Liu, T.; Duan, T.; Wu, Y.; Cui, J. Tetherin Suppresses Type I Interferon Signaling by Targeting MAVS for NDP52-Mediated Selective Autophagic Degradation in Human Cells. Mol. Cell. 2017, 68, 308-322.e4. [CrossRef]

29. Nakhaei, P.; Mesplede, T.; Solis, M.; Sun, Q.; Zhao, T.; Yang, L.; Chuang, T.H.; Ware, C.F.; Lin, R.; Hiscott, J. The E3 ubiquitin ligase Triad3A negatively regulates the RIG-I/MAVS signaling pathway by targeting TRAF3 for degradation. PLoS Pathog. 2009, 5, e1000650. [CrossRef] [PubMed]

30. Sun, Y.; Zheng, H.; Yu, S.; Ding, Y.; Wu, W.; Mao, X.; Liao, Y.; Meng, C.; Ur Rehman, Z.; Tan, L.; et al. Newcastle Disease Virus V Protein Degrades Mitochondrial Antiviral Signaling Protein To Inhibit Host Type I Interferon Production via E3 Ubiquitin Ligase RNF5. J. Virol. 2019, 93, e00322-19. [CrossRef] [PubMed]

31. Zhou, X.Y.; Wang, Y.; Zhu, J.; Miao, Q.-H.; Zhu, L.Q.; Zhan, S.H.; Wang, G.J.; Liu, G.Q. First report of peste des petits ruminants virus lineage II in Hydropotes inermis, China. Transbound. Emerg. Dis. 2018, 65, e205-e209. [CrossRef]

32. Pruvot, M.; Fine, A.E.; Hollinger, C.; Strindberg, S.; Damdinjav, B.; Buuveibaatar, B.; Chimeddorj, B.; Bayandonoi, G.; Khishgee, B.; Sandag, B.; et al. Outbreak of Peste des Petits Ruminants among Critically Endangered Mongolian Saiga and Other Wild Ungulates, Mongolia, 2016-2017. Emerg. Infect. Dis. 2020, 26, 51-62. [CrossRef]

33. Shahriari, R.; Khodakaram-Tafti, A.; Mohammadi, A. Molecular characterization of Peste des Petits ruminants virus isolated from four outbreaks occurred in southern Iran. BMC Vet. Res. 2019, 15, 177. [CrossRef]

34. Rojas, J.M.; Moreno, H.; Valcarcel, F.; Pena, L.; Sevilla, N.; Martin, V. Vaccination with recombinant adenoviruses expressing the peste des petits ruminants virus $\mathrm{F}$ or $\mathrm{H}$ proteins overcomes viral immunosuppression and induces protective immunity against PPRV challenge in sheep. PLoS ONE 2014, 9, e101226. [CrossRef]

35. Li, P.; Zhu, Z.; Zhang, X.; Dang, W.; Li, L.; Du, X.; Zhang, M.; Wu, C.; Xue, Q.; Liu, X.; et al. The Nucleoprotein and Phosphoprotein of Peste des Petits Ruminants Virus Inhibit Interferons Signaling by Blocking the JAK-STAT Pathway. Viruses 2019, 11, 7. [CrossRef] [PubMed]

36. Zhu, Z.; Li, P.; Yang, F.; Cao, W.; Zhang, X.; Dang, W.; Ma, X.; Tian, H.; Zhang, K.; Zhang, M.; et al. Peste des Petits Ruminants Virus Nucleocapsid Protein Inhibits Beta Interferon Production by Interacting with IRF3 To Block Its Activation. J. Virol. 2019, 93, 16. [CrossRef] [PubMed]

37. Kell, A.M.; Hemann, E.A.; Turnbull, J.B.; Gale, M., Jr. RIG-I-like receptor activation drives type I IFN and antiviral signaling to limit Hantaan orthohantavirus replication. PLoS Pathog. 2020, 16, e1008483. [CrossRef] 
38. You, F.; Sun, H.; Zhou, X.; Sun, W.; Liang, S.; Zhai, Z.; Jiang, Z. PCBP2 mediates degradation of the adaptor MAVS via the HECT ubiquitin ligase AIP4. Nat. Immunol. 2009, 10, 1300-1308. [CrossRef] [PubMed]

39. Zhou, X.; You, F.; Chen, H.; Jiang, Z. Poly(C)-binding protein 1 (PCBP1) mediates housekeeping degradation of mitochondrial antiviral signaling (MAVS). Cell Res. 2012, 22, 717-727. [CrossRef] [PubMed]

40. Qin, Y.; Xue, B.; Liu, C.; Wang, X.; Tian, R.; Xie, Q.; Guo, M.; Li, G.; Yang, D.; Zhu, H. NLRX1 Mediates MAVS Degradation To Attenuate the Hepatitis C Virus-Induced Innate Immune Response through PCBP2. J. Virol. 2017, 91, 23. [CrossRef]

41. Zhang, X.; Zhu, C.; Wang, T.; Jiang, H.; Ren, Y.; Zhang, Q.; Wu, K.; Liu, F.; Liu, Y.; Wu, J. GP73 represses host innate immune response to promote virus replication by facilitating MAVS and TRAF6 degradation. PLoS Pathog. 2017, 13, e1006321. [CrossRef] [PubMed]

42. Ding, S.; Zhu, S.; Ren, L.; Feng, N.; Song, Y.; Ge, X.; Li, B.; Flavell, R.A.; Greenberg, H.B. Rotavirus VP3 targets MAVS for degradation to inhibit type III interferon expression in intestinal epithelial cells. Elife 2018, 7, e39494. [CrossRef] [PubMed]

43. Yu, L.; Zhang, X.; Wu, T.; Su, J.; Wang, Y.; Wang, Y.; Ruan, B.; Niu, X.; Wu, Y. Avian infectious bronchitis virus disrupts the melanoma differentiation associated gene 5 (MDA5) signaling pathway by cleavage of the adaptor protein MAVS. BMC Vet. Res. 2017, 13, 332. [CrossRef]

44. Linjie, L.; Xiaoling, S.; Xiaoxia, M.; Xin, C.; Ali, A.; Jialin, B. Peste des petits ruminants virus non-structural C protein inhibits the induction of interferon- $\beta$ by potentially interacting with MAVS and RIG-I. Virus Genes 2021, 57, 60-71. [CrossRef]

45. Libeau, G.; Diallo, A.; Parida, S. Evolutionary genetics underlying the spread of peste des petits ruminants virus. Anim. Front. 2014, 4, 14-20. [CrossRef] 